\title{
THE SPECTRA OF THE SURFACE MARYLAND MODEL FOR ALL PHASES
}

\author{
WENCAI LIU
}

(Communicated by Michael Hitrik)

\begin{abstract}
We study the discrete Schrödinger operators $H_{\lambda, \alpha, \theta}$ on $\ell^{2}\left(\mathbb{Z}^{d+1}\right)$ with surface potential of the form $V(n, x)=\lambda \delta(x) \tan \pi(\alpha \cdot n+\theta)$, and $H_{\lambda, \alpha, \theta}^{+}$ on $\ell^{2}\left(\mathbb{Z}^{d} \times \mathbb{Z}_{+}\right)$with the boundary condition $\psi_{(n,-1)}=\lambda \tan \pi(\alpha \cdot n+\theta) \psi_{(n, 0)}$, where $\alpha \in \mathbb{R}^{d}$ is rationally independent. We show that the spectra of $H_{\lambda, \alpha, \theta}$ and $H_{\lambda, \alpha, \theta}^{+}$are $(-\infty, \infty)$ for all parameters. We can also determine the absolutely continuous spectra and Hausdorff dimension of the spectral measures if $d=1$.
\end{abstract}

\section{INTRODUCTION}

In this paper, we study the discrete Schrödinger operators with surface potential. Let $d \geq 1$ be given and $\mathbb{Z}_{+}=\{0,1,2, \cdots\}$. We denote the points in $\mathbb{Z}^{d+1}$ (or $\left.\mathbb{Z}_{+}^{d+1}=\mathbb{Z}^{d} \times \mathbb{Z}_{+}\right)$by $(n, x)$, where $n \in \mathbb{Z}^{d}, x \in \mathbb{Z}$ (or $n \in \mathbb{Z}^{d}, x \in \mathbb{Z}_{+}$).

Given $y=\left(y_{1}, y_{2}, \cdots, y_{k}\right) \in \mathbb{Z}^{k}$ with $k \geq 1$, denote by $\|y\|_{k}=\sum_{j=1}^{k}\left|y_{j}\right|$.

Now we introduce two self-adjoint operators $H_{\lambda, \alpha, \theta}$ and $H_{\lambda, \alpha, \theta}^{+}$. The operator $H_{\lambda, \alpha, \theta}$ acts on $\ell^{2}\left(\mathbb{Z}^{d+1}\right)$ as

$$
\left(H_{\lambda, \alpha, \theta} \psi\right)_{(n, x)}=\sum_{\left\|\left(n-n^{\prime}, x-x^{\prime}\right)\right\|_{d+1}=1} \psi_{\left(n^{\prime}, x^{\prime}\right)}+\lambda \delta(x) \tan \pi(\alpha \cdot n+\theta) \psi_{(n, x)},
$$

where $\lambda \neq 0$ is the coupling, $\alpha \in \mathbb{R}^{d}$ is the frequency, and $\theta$ is the phase. In order to make the potential well defined, we always assume $\theta$ satisfies the following condition:

$$
k \cdot \alpha+\theta \neq \frac{1}{2} \quad \bmod \mathbb{Z} \text { for any } k \in \mathbb{Z}^{d} .
$$

The other operator $H_{\lambda, \alpha, \theta}^{+}$on $\ell^{2}\left(\mathbb{Z}^{d} \times \mathbb{Z}_{+}\right)$is given by the following equation:

$$
\left(H_{\lambda, \alpha, \theta}^{+} \psi\right)_{(n, x)}= \begin{cases}\sum_{\left\|\left(n-n^{\prime}, x-x^{\prime}\right)\right\|_{d+1}=1} \psi_{\left(n^{\prime}, x^{\prime}\right)}, & \text { if } x>0 \\ \psi_{(n, 1)}+\sum_{\left\|n^{\prime}-n\right\|_{d}=1} \psi_{\left(n^{\prime}, 0\right)}+\lambda \tan \pi(\alpha \cdot n+\theta) \psi_{(n, 0)}, & \text { if } x=0\end{cases}
$$

Whenever the meaning is clear within the context, we will drop the dependence on parameters $\lambda, \alpha, \theta$ and $d$. Thus we write $H$ or $H_{\theta}$ for $H_{\lambda, \alpha, \theta}$, and $\left\|n^{\prime}-n\right\|$ for $\left\|n^{\prime}-n\right\|_{d}$

Received by the editors November 18, 2015 and, in revised form, January 11, 2016.

2010 Mathematics Subject Classification. Primary 11F72, 37A30, 47A10. 
Recall that the Maryland model is given by

$$
\left(h_{\lambda, \alpha, \theta} \psi\right)_{n}=\sum_{\left\|n^{\prime}-n\right\|=1} \psi_{n^{\prime}}+\lambda \tan \pi(\alpha \cdot n+\theta) \psi_{n},
$$

with $n, n^{\prime} \in \mathbb{Z}^{d}$. It has been heavily studied in [4, 6, 13. In particular, for $d=1$, Jitomirskaya-Liu has obtained the complete description of the three spectral types of the Maryland model [10].

Now we want to say more about the surface multidimensional operators. Let $H_{0}$ be the discrete Laplacian on $\ell^{2}\left(\mathbb{Z}^{d+1}\right)$, and $H_{0}^{+}$be the discrete Laplacian on $\ell^{2}\left(\mathbb{Z}_{+}^{d+1}\right)$ with the Dirichlet boundary condition. More precisely,

$$
\left(H_{0} \psi\right)_{y}=\sum_{\left\|y^{\prime}-y\right\|=1} \psi_{y^{\prime}}
$$

where $y, y^{\prime} \in \mathbb{Z}^{d+1}$, and

$$
\left(H_{0}^{+} \psi\right)_{(n, x)}=\sum_{\left\|\left(n^{\prime}-x^{\prime}, n-x\right)\right\|=1} \psi_{\left(n^{\prime}, x^{\prime}\right)}
$$

with $\psi_{(n,-1)}=0$, where $n \in \mathbb{Z}^{d}$ and $x \in \mathbb{Z}_{+}$.

Consider the operator $H$. The support of the potential is on the hyperplane $\mathbb{Z}^{d}$ of the space $\mathbb{Z}^{d+1}$. For the other one, $H^{+}$, it is the discrete Laplacian on $\ell^{2}\left(\mathbb{Z}_{+}^{d+1}\right)$ with the boundary condition $\psi_{(n,-1)}=\lambda \tan \pi(\alpha \cdot n+\theta) \psi_{(n, 0)}$. Thus it is natural to call both operators $H$ and $H_{+}$the surface Maryland model.

Denote by $\sigma(H)$ and $\sigma_{a c}(H)$ the spectrum, and absolutely continuous spectrum of the self-adjoint operator $H$. We recall that $\sigma\left(H_{0}\right)=[-2(d+1), 2(d+1)]\left(\sigma\left(H_{0}^{+}\right)=\right.$ $[-2(d+1), 2(d+1)])$ and the spectrum of $H_{0}\left(H_{0}^{+}\right)$is purely absolutely continuous. Denote by $c_{d}=2(d+1)$.

We recall that $\alpha=\left(\alpha_{1}, \alpha_{2}, \cdots, \alpha_{d}\right)$ is rationally independent if for any choice of integers $\left(k_{1}, k_{2}, \cdots, k_{d}\right) \neq 0$,

$$
\sum_{j=1}^{d} k_{j} \alpha_{j} \notin \mathbb{Z} .
$$

In the rest of the paper, we always assume $\lambda \neq 0, \alpha=\left(\alpha_{1}, \alpha_{2}, \cdots, \alpha_{d}\right)$ is rationally independent and $\theta$ satisfies condition (2). We will often use "all $\lambda(\alpha, \theta)$ ", meaning "all $\lambda(\alpha, \theta)$ as above".

Now we start to state some known results.

Theorem 1.1 ([1, [7]). Let $H_{\lambda, \alpha, \theta}$ and $H_{\lambda, \alpha, \theta}^{+}$be given by (10) and (3) respectively. Then for all parameters $(\lambda, \alpha, \theta),\left[-c_{d}, c_{d}\right] \subset \sigma_{a c}\left(H_{\lambda, \alpha, \theta}\right), \sigma_{a c}\left(H_{\lambda, \alpha, \theta}^{+}\right)$, and $H_{\lambda, \alpha, \theta}$, $H_{\lambda, \alpha, \theta}^{+}$has purely absolutely continuous spectrum on the interval $\left(-c_{d}, c_{d}\right)$.

We say $\alpha \in \mathbb{R}^{d}$ satisfies a Diophantine condition if there exists $\kappa>0$ and $\tau>0$, such that

$$
\|k \cdot \alpha\|_{\mathbb{R} / \mathbb{Z}}>\kappa\|k\|^{-\tau} \text { for any } k \in \mathbb{Z}^{d} \backslash\{0\},
$$

where $\|x\|_{\mathbb{R} / \mathbb{Z}}=\min _{j}|x-j|$.

It is to be believed that $H$ and $H^{+}$have purely singular spectra outside the set $\left[-c_{d}, c_{d}\right]$. Khoruzenko and Pastur [1] have proved this for $\alpha$ satisfying a Diophantine condition. 
Theorem 1.2 (11). Assume frequency $\alpha$ satisfies a Diophantine condition (41). Then $\sigma\left(H_{\lambda, \alpha, \theta}\right)=(-\infty, \infty)$ and $H_{\lambda, \alpha, \theta}$ only has pure point on $(-\infty, \infty) \backslash\left[-c_{d}, c_{d}\right]$. On this set, the eigenvalues are simple and the corresponding eigenfunctions decay exponentially. These properties also apply to the operator $H_{\lambda, \alpha, \theta}^{+}$.

For the surface Maryland model, one of the basic ideas is to integrate the $x$ variable and to reduce the $(d+1)$-dimensional spectral problem to a $d$-dimensional problem. This technology has been heavily developed by use of Green function in 7, 9, 11. This technology can also be extended to the generalized surface Maryland model [1,2]. In this paper, we also use this basic technology to analyze the spectra of the surface Maryland model. However, we shed new light on the subject.

Our first result is to obtain the explicit formula of the spectra of $H_{\lambda, \alpha, \theta}$ and $H_{\lambda, \alpha, \theta}^{+}$.

Theorem 1.3. Suppose $\alpha$ is rationally independent and $\lambda \neq 0$; then $\sigma\left(H_{\lambda, \alpha, \theta}\right)=$ $\sigma\left(H_{\lambda, \alpha, \theta}^{+}\right)=(-\infty,+\infty)$ for all $\theta$.

Remark. From Theorems 1.1 and 1.2, we directly know that Theorem 1.3 holds for Diophantine $\alpha$. A Diophantine condition is necessary for Theorem 1.2 when we solve the cohomological equation. Our aim is to prove Theorem 1.3 for non-Diophantine $\alpha$.

Secondly we study the absolutely continuous spectrum. By Theorem 1.1, $H$ $\left(H^{+}\right)$has purely absolutely continuous spectrum in the interval $\left(-c_{d}, c_{d}\right)$. It was not known if $H\left(H^{+}\right)$had some absolutely continuous component in the remaining interval $\mathbb{R} \backslash\left[-c_{d}, c_{d}\right]$. It is believed that $H\left(H^{+}\right)$has no absolutely continuous component in $\mathbb{R} \backslash\left[-c_{d}, c_{d}\right]$. In this paper, we solve the problem for $d=1$. We construct an arithmetically defined measure zero set (actually Hausdorff dimension zero) that supports spectral measures in the regime $\mathbb{R} \backslash\left[-c_{d}, c_{d}\right]$, which directly implies the following theorem.

Theorem 1.4. Let $H_{\lambda, \alpha, \theta}$ and $H_{\lambda, \alpha, \theta}^{+}$be given by (11) and (3) respectively with $d=1$. Suppose $\alpha \in \mathbb{R} \backslash \mathbb{Q}$; then $\sigma_{a c}\left(H_{\lambda, \alpha, \theta}\right)=\sigma_{a c}\left(H_{\lambda, \alpha, \theta}^{+}\right)=[-4,4]$ and $H_{\lambda, \alpha, \theta}$, $H_{\lambda, \alpha, \theta}^{+}$has purely absolutely continuous spectra on $(-4,4)$ for all $\theta$. Furthermore, the spectral measures of both operators are of Hausdorff dimension one restricted to $(-4,4)$ and of Hausdorff dimension zero restricted to $\mathbb{R} \backslash[-4,4]$ for all $\theta$.

Remark 1.5. The fact that the spectral measures restricted to $(-4,4)$ are of Hausdorff dimension one is trivial because $H_{\lambda, \alpha, \theta}$ and $H_{\lambda, \alpha, \theta}^{+}$have purely absolutely continuous spectra on $(-4,4)$.

We will prove Theorems 1.3 and 1.4 in two steps. First, we will reduce the $(d+1)$-dimensional spectral problem to a $d$-dimensional problem in $\S 2$. Second, we will study the $d$-dimensional problem using the ideas in paper [10] ( $§ 3$ and $\S 4$ ), in which the authors deal with the Maryland model.

\section{GREen FUnCTION AND DIMENSION REDUCTION}

Because our arguments are almost the same for the operators $H$ and $H^{+}$, we concentrate on $H$ in the following discussion, and only point out the difference for $H^{+}$.

In this section, we carry out the dimension reduction for the surface Maryland model, and lay the ground work for our main results. We first consider the operator 
$H$. We should point out that all the contents in this section are from [1,7, 11]. For convenience, we rewrote it here.

Denote by $G\left(X_{1} ; X_{2}\right)$ and $G_{0}\left(X_{1}-X_{2}\right)$ the Green functions of the operators $H$ and $H_{0}$ respectively, i.e., the infinite dimensional matrices $(H-z I)^{-1}$ and $\left(H_{0}-\right.$ $z I)^{-1}$ with $\Im z \neq 0$. Then, using the resolvent identity, one has

$$
G\left(X_{1} ; X_{2}\right)=G_{0}\left(X_{1}-X_{2}\right)-\sum_{\eta \in \mathbb{Z}^{d}} G_{0}\left(n_{1}-\eta, x_{1}\right) \lambda v(\eta) G\left(\eta, 0 ; X_{2}\right),
$$

where $X_{1}=\left(n_{1}, x_{1}\right) \in \mathbb{Z}^{d+1}, X_{2}=\left(n_{2}, x_{2}\right) \in \mathbb{Z}^{d+1}$ and $v(\eta)=\tan \pi(\alpha \cdot \eta+\theta)$. For simpleness, let $v\left(v^{-1}\right)$ be the multiplication operator by $v(\eta)(1 / v(\eta))$ on $\ell^{2}\left(\mathbb{Z}^{d}\right)$.

Setting $x_{1}=0$ in (5), we find that the function $g_{x_{2}}\left(n_{1} ; n_{2}\right)=G\left(n_{1}, 0 ; n_{2}, x_{2}\right)$ satisfies

$$
g_{x_{2}}\left(n_{1} ; n_{2}\right)=g_{0, x_{2}}\left(n_{1}-n_{2}\right)-\sum_{\eta \in \mathbb{Z}^{d}} \Gamma_{0}\left(n_{1}-\eta\right) \lambda v(\eta) g_{x_{2}}\left(\eta ; n_{2}\right),
$$

where $g_{0, x_{2}}(\eta)=G_{0}\left(\eta,-x_{2}\right)$ and $\Gamma_{0}(\eta)=G_{0}(\eta, 0)$ with $\eta \in \mathbb{Z}^{d}$. Next we always regard $\Gamma_{0}(\eta)$ as an operator on $\mathbb{Z}^{d}$.

Fixing $x_{2}$, equation (6) becomes a problem in dimension $d$, and has a symbolic solution $g_{x_{2}}=\left(I+\Gamma_{0} \lambda v\right)^{-1} g_{0, x_{2}}$. Combining with (5), we have

$$
\begin{aligned}
G\left(X_{1} ; X_{2}\right) & =G\left(n_{1}, x_{1} ; n_{2}, x_{2}\right) \\
& =G_{0}\left(X_{1}-X_{2}\right)-\sum_{\eta_{1}, \eta_{2} \in \mathbb{Z}^{d}} G_{0}\left(n_{1}-\eta_{1}, x_{1}\right) T\left(\eta_{1}, \eta_{2}\right) G_{0}\left(\eta_{2}-n_{2},-x_{2}\right),
\end{aligned}
$$

where $T=\lambda v\left(I+\Gamma_{0} \lambda v\right)^{-1}=\left(\lambda^{-1} v^{-1}+\Gamma_{0}\right)^{-1}$.

Notice that the above derivation of equation (77) is only symbolic, because generally, the operator $\left(I+\Gamma_{0} \lambda v\right)^{-1}$ is not well defined. However, the following theorem shows (7) is true in fact.

Theorem 2.1 (pp. 116-118, 11]). Let $U$ denote the multiplication by $e^{2 \pi i \alpha \cdot n}$ on $\ell^{2}\left(\mathbb{Z}^{d}\right)$ and $\delta=e^{2 \pi i \theta}$. If $\Im z \cdot \lambda<0$, then equation (7) holds, where $T$ can be written as

$$
T=\lambda(1-\delta U)(I-\delta C U)^{-1}\left(\lambda \Gamma_{0}-i I\right)^{-1},
$$

where

$$
C=\left(\lambda \Gamma_{0}-i I\right)^{-1}\left(\lambda \Gamma_{0}+i I\right) .
$$

Proof. Suppose we can prove $\lambda \Gamma_{0}-i I$ is invertible and $\|C\|<1$; then equation (8) is well defined, and (7) holds by direct computation. Now we start to prove the facts by using Fourier transformation. Given a vector $f_{n}$ in $\ell^{2}\left(\mathbb{Z}^{b}\right)$ with $b=d$ or $b=d+1$, define an $L^{2}\left(\mathbb{T}^{b}\right)$ function by the following equation:

$$
\hat{f}(y)=\sum_{n \in \mathbb{Z}^{b}} f_{n} e^{-2 \pi i n \cdot y} .
$$

Recall that $\mathbb{T}=\mathbb{R} / \mathbb{Z}$. We let $\hat{f}(y)$ be the momentum representation of vector $f_{n}$. Under the Fourier transformation, $H_{0}$ becomes a multiplication operator on $L^{2}\left(\mathbb{T}^{d+1}\right)$ and is denoted by $\hat{H}_{0}$. More precisely,

$$
\left(\hat{H}_{0} \hat{f}\right)(y)=\left(\sum_{j=1}^{d+1} 2 \cos 2 \pi y_{j}\right) \hat{f}(y) .
$$


Thus in momentum representation, Green function $G_{0}$ has the following simple form:

$$
\left(\hat{G}_{0} \hat{f}\right)(y)=\left(\frac{1}{\sum_{j=1}^{d+1} 2 \cos 2 \pi y_{j}-z}\right) \hat{f}(y),
$$

with $y \in \mathbb{T}^{d+1}$, and the operator $\Gamma_{0}$ becomes

$$
\left(\hat{\Gamma}_{0} \hat{f}\right)(y)=\hat{\Gamma}_{0}(y) \hat{f}(y),
$$

where

$$
\hat{\Gamma}_{0}(y)=\int_{\mathbb{T}} \frac{1}{\sum_{j=1}^{d+1} 2 \cos 2 \pi y_{j}-z} d y_{d+1},
$$

with $y \in \mathbb{T}^{d}$. Notice that $\Im \lambda \hat{\Gamma}_{0}(y)<0$; then $\lambda \Gamma_{0}-i I$ is invertible (because of $\Im z \cdot \lambda<0)$. Furthermore, $C$ also becomes a multiplication operator in momentum representation,

$$
(\hat{C} \hat{f})(y)=\frac{\lambda \hat{\Gamma}_{0}(y)+i}{\lambda \hat{\Gamma}_{0}(y)-i} \hat{f}(y) .
$$

This yields $\|C\|<1$.

Now we turn to the operator $H^{+}$. If an operator $A$ generates from $H$, then denote $A^{+}$the corresponding operator generated from $H^{+}$. For example, $G^{+}=$ $\left(H^{+}-z I\right)^{-1}$. Using a similar argument as before, one has

$$
G^{+}\left(X_{1} ; X_{2}\right)=G_{0}^{+}\left(X_{1} ; X_{2}\right)-\sum_{\eta \in \mathbb{Z}^{d}} G_{0}^{+}\left(n_{1}, x_{1} ; \eta, 0\right) v(\eta) G^{+}\left(\eta, 0 ; X_{2}\right),
$$

where $X_{1}=\left(n_{1}, x_{1}\right) \in \mathbb{Z}_{+}^{d+1}, X_{2}=\left(n_{2}, x_{2}\right) \in \mathbb{Z}_{+}^{d+1}$ and $v(\eta)=\lambda \tan \pi(\alpha \cdot \eta+\theta)$. We should point out that $G_{0}^{+}\left(n_{1}, x_{1} ; n_{2}, x_{2}\right)$ depends on $n_{1}-n_{2}, x_{1}, x_{2}$. This is different from $G_{0}\left(n_{1}, x_{1} ; n_{2}, x_{2}\right)$, which only depends on $n_{1}-n_{2}, x_{1}-x_{2}$.

Setting $x_{1}=0$ in (10), we find that the function $g_{x_{2}}^{+}\left(n_{1} ; n_{2}\right)=G^{+}\left(n_{1}, 0 ; n_{2}, x_{2}\right)$ satisfies

$$
g_{x_{2}}^{+}\left(n_{1} ; n_{2}\right)=g_{0, x_{2}}^{+}\left(n_{1} ; n_{2}\right)-\sum_{\eta \in \mathbb{Z}^{d}} \Gamma_{0}^{+}\left(n_{1}-\eta\right) v(\eta) g_{x_{2}}^{+}\left(\eta ; n_{2}\right)
$$

where $g_{0, x_{2}}^{+}\left(n_{1} ; n_{2}\right)=G_{0}^{+}\left(n_{1}, 0 ; n_{2}, x_{2}\right)$ and $\Gamma_{0}^{+}(\eta)=G_{0}^{+}(\eta, 0 ; 0,0)$ with $\eta \in \mathbb{Z}^{d}$.

Fixing $x_{2}$, equation (11) becomes a problem in dimension $d$, and has a symbolic solution $g_{x_{2}}^{+}=\left(I+\Gamma_{0}^{+} v\right)^{-1} g_{0, x_{2}}^{+}$. Combining with (10), we have

$$
\begin{aligned}
G^{+}\left(X_{1} ; X_{2}\right) & =G^{+}\left(n_{1}, x_{1} ; n_{2}, x_{2}\right) \\
& =G_{0}^{+}\left(n_{1}, x_{1} ; n_{2}, x_{2}\right)-\sum_{\eta_{1}, \eta_{2} \in \mathbb{Z}^{d}} G_{0}^{+}\left(n_{1}, x_{1} ; \eta_{1}, 0\right) T^{+}\left(\eta_{1}, \eta_{2}\right) G_{0}^{+}\left(\eta_{2}, 0 ; n_{2}, x_{2}\right),
\end{aligned}
$$

where $T^{+}=v\left(I+\Gamma_{0}^{+} v\right)^{-1}=\left(v^{-1}+\Gamma_{0}^{+}\right)^{-1}$.

We should say more about the operator $\Gamma_{0}^{+}$. In momentum representation, $\Gamma_{0}^{+}$ has the following form [7]:

$$
\left(\hat{\Gamma}_{0}^{+} \hat{f}\right)(y)=-r(y, z) \hat{f}(y),
$$


where $r(y, z)$ is the root of the quadratic equation

$$
X+\frac{1}{X}+\sum_{j}^{d} 2 \cos 2 \pi y_{j}=z
$$

with $|r(y, z)|<1$.

By a similar proof as above, we have the following theorem.

Theorem 2.2. Suppose $\Im z \cdot \lambda<0$; then equation (12) holds and $T^{+}$can be written as

$$
T^{+}=\lambda(1-\delta U)\left(I-\delta C^{+} U\right)^{-1}\left(\lambda \Gamma_{0}^{+}-i I\right)^{-1},
$$

where

$$
C^{+}=\left(\lambda \Gamma_{0}^{+}-i I\right)^{-1}\left(\lambda \Gamma_{0}^{+}+i I\right) .
$$

Remark 2.3. Notice that for $z \in \mathbb{C} \backslash\left[-c_{d}, c_{d}\right]$, the operators $\Gamma_{0}$ and $\Gamma_{0}^{+}$are well defined.

\section{The spectra of the surface Maryland Model}

In this section, we will prove that for the surface Maryland model $\sigma\left(H_{\lambda, \alpha, \theta}\right)=$ $(-\infty, \infty)$ and $\sigma\left(H_{\lambda, \alpha, \theta}^{+}\right)=(-\infty, \infty)$. From Theorem 1.2, one has that for DC $\alpha$, $\sigma\left(H_{\lambda, \alpha, \theta}\right)=(-\infty, \infty), \sigma\left(H_{\lambda, \alpha, \theta}^{+}\right)=(-\infty, \infty)$ for all $\lambda \neq 0$ and $\theta$. Our main work in this section is to show that the spectrum $\sigma\left(H_{\lambda, \alpha, \theta}\right)$ does not depend on $\theta$.

Following the discussion of unbounded operators in section VIII [12, it suffices to show the self-adjoint operators $H_{\lambda, \alpha, \theta}$ are continuous in the norm resolvent sense with respect to $\theta$. Combining with the specific formula of the Green function of the surface Maryland model (Theorem 2.1 and Theorem 2.2), we can obtain the results. In [10, the authors have already used this method to show that the spectrum of the Maryland model does not depend on phase $\theta$.

Definition 3.1. Let $B_{n}, n=1,2, \cdots$ and $B$ be self-adjoint operators; then $B_{n}$ is said to converge to $B$ in the norm resolvent sense if $G_{z}\left(B_{n}\right) \rightarrow G_{z}(B)$ in norm for all $z$ with $\Im z \neq 0$, where $G_{z}(A)=(A-z I)^{-1}$.

Theorem 3.2 (Theorem VIII.24, [12]). Let $B_{n}, n=1,2, \cdots$ and $B$ be self-adjoint operators, and $B_{n}$ converge to $B$ in the norm resolvent sense. Then for any $E \in$ $\sigma(B)$, there exists $E_{n} \in \sigma\left(B_{n}\right)$ such that $E_{n} \rightarrow E$.

Theorem 3.3. The spectrum of the surface Maryland model does not depend on phase $\theta$. More precisely, neither $\sigma\left(H_{\lambda, \alpha, \theta}\right)$ nor $\sigma\left(H_{\lambda, \alpha, \theta}^{+}\right)$depends on phase $\theta$.

Proof. For the two given phases $\theta_{1}$ and $\theta_{2}$, there exists some sequence $j_{k} \in \mathbb{Z}^{d}$ such that

$$
\lim _{k \rightarrow \infty}\left\|\theta_{1}+j_{k} \cdot \alpha-\theta_{2}\right\|_{\mathbb{R} / \mathbb{Z}} \rightarrow 0
$$

since $\alpha$ is rationally independent.

Let $B_{k}=H_{\lambda, \alpha, \theta_{1}+j_{k} \cdot \alpha}\left(B_{k}=H_{\lambda, \alpha, \theta_{1}+j_{k} \cdot \alpha}^{+}\right)$and $B=H_{\lambda, \alpha, \theta_{2}}\left(B=H_{\lambda, \alpha, \theta_{2}}^{+}\right)$. We must have $B_{k}$ converge to $B$ in the norm resolvent sense. Indeed, if $\lambda$. $\Im z<0$, this is easy to see by (77) and (8) (12) and (13)); if $\lambda \cdot \Im z>0$, this is also true by the fact $H_{\lambda, \alpha, \theta}=H_{-\lambda,-\alpha,-\theta}\left(H_{\lambda, \alpha, \theta}^{+}=H_{-\lambda,-\alpha,-\theta}^{+}\right)$.

Applying Theorem 3.2, for any $E \in \sigma\left(H_{\lambda, \alpha, \theta_{2}}\right)\left(\sigma\left(H_{\lambda, \alpha, \theta_{2}}^{+}\right)\right)$, there exists $E_{k} \in$ $\sigma\left(B_{k}\right)$ such that $E_{k} \rightarrow E$. Clearly, the spectrum of operator $B_{k}$ does not depend 
on $k$, i.e., $\sigma\left(B_{k}\right)=\sigma\left(H_{\lambda, \alpha, \theta_{1}}\right)\left(\sigma\left(B_{k}\right)=\sigma\left(H_{\lambda, \alpha, \theta_{1}}^{+}\right)\right)$because the shift operator is a unitary operator. Thus we must have

$$
\sigma\left(H_{\lambda, \alpha, \theta_{2}}\right) \subseteq \sigma\left(H_{\lambda, \alpha, \theta_{1}}\right)
$$

and

$$
\sigma\left(H_{\lambda, \alpha, \theta_{2}}^{+}\right) \subseteq \sigma\left(H_{\lambda, \alpha, \theta_{1}}^{+}\right) .
$$

This implies the theorem since $\theta_{1}$ and $\theta_{2}$ are arbitrary.

The proof of Theorem 1.3. To avoid repetition, we only need to prove the theorem for operator $H$. Denote by $\mathbb{E}\{\cdot\}$ the average value over $\mathbb{T}$. Since $\|C\|<1$, one has

$$
(I-\delta C U)^{-1}=I+\sum_{k=1}^{\infty}(\delta C U)^{k} .
$$

Notice that $\delta U=U \delta$ and $\delta C=C \delta$. We have

$$
(I-\delta C U)^{-1}=I+\sum_{k=1}^{\infty} \delta^{k}(C U)^{k} .
$$

Putting (8) into (7) and integrating over $\mathbb{T}$ with respect to the variable $\theta$, one has that $\mathbb{E}\{G\}$ does not depend on $\alpha$.

Suppose Theorem 1.3 does not hold. Then by Theorem 3.3 there exist some $\alpha_{1} \in \mathbb{R}^{d}$ and an interval $I$ such that $I \subset(-\infty, \infty) \backslash \sigma\left(H_{\lambda, \alpha_{1}, \theta}\right)$ for all $\theta$. Thus for any given vectors $\delta_{y}, y \in \mathbb{Z}^{d+1}$, the spectral measure $\mu_{\theta, \alpha_{1}, y}$ determined by

$$
\left(\left(H_{\lambda, \alpha_{1}, \theta}-z I\right)^{-1} \delta_{y}, \delta_{y}\right)=\int \frac{d \mu_{\theta, \alpha_{1}, y}(\phi)}{\phi-z}
$$

satisfies

$$
\mu_{\theta, \alpha_{1}, y}(I)=0 \text { for all } \theta .
$$

Using the fact that $\mathbb{E}\{G\}$ does not depend on $\alpha$ and (14), one has

$$
\int_{\mathbb{T}} \mu_{\theta, \alpha, y}(I) d \theta=\int_{\mathbb{T}} \mu_{\theta, \alpha_{1}, y}(I) d \theta=0
$$

for any $\alpha \in \mathbb{R}^{d}$ and $y \in \mathbb{Z}^{d+1}$. Combining with the fact that $\sigma\left(H_{\lambda, \alpha, \theta}\right)$ does not depend on $\theta$, we have

$$
I \subset(-\infty, \infty) \backslash \sigma\left(H_{\lambda, \alpha, \theta}\right)
$$

for all $\alpha \in \mathbb{R}^{d}$. This is a contradiction to Theorems 1.1 and 1.2 .

\section{The SPeCtral MeAsures For $d=1$}

We always assume $E \in(-\infty, \infty) \backslash\left[-c_{d}, c_{d}\right]$ below.

Let

$$
\mathcal{P}_{b}=\left\{\left\{\psi_{y}\right\}_{y \in \mathbb{Z}^{b}}:\left|\psi_{y}\right| \leq C\left(1+\|y\|^{C}\right) \text { for some constant } C\right\},
$$

where $b=d$ or $b=d+1$. Usually, we call elements in $\mathcal{P}_{b}$ polynomially bounded vectors.

Theorem 4.1. If the equation

$$
v(n)^{-1} \varphi_{n}+\sum_{\eta \in \mathbb{Z}^{d}} \lambda \Gamma_{0}(n-\eta) \varphi_{\eta}=0
$$


with $n \in \mathbb{Z}^{d}$ has a non-zero polynomially bounded solution $\varphi$, then

$$
\psi_{(n, x)}=\sum_{\eta \in \mathbb{Z}^{d}} G_{0}(n-\eta, x) \varphi_{\eta}
$$

solves equation (11) and $\psi_{(n, x)}$ is non-zero and polynomially bounded. If equation (11) has a non-zero polynomially bounded solution, define

$$
\varphi_{n}=\sum_{\eta \in \mathbb{Z}^{d}} \Gamma_{0}^{-1}(n-\eta) \psi_{(\eta, 0)} .
$$

Then $\varphi_{n}$ solves equation (15) and $\varphi_{n}$ is non-zero and polynomially bounded.

Notice that for $E \in(-\infty, \infty) \backslash\left[-c_{d}, c_{d}\right]$, the operator $\Gamma_{0}$ is well defined and

$$
\left|\Gamma_{0}^{-1}(n)\right|,\left|\Gamma_{0}(n)\right| \leq e^{-t|| n||}
$$

for some $t>0\left(t\right.$ depends on the distance between $E$ and $\left.\left[-c_{d}, c_{d}\right]\right)$. Similarly, for some $t>0$,

with $n \in \mathbb{Z}^{d}$.

$$
\left|G_{0}(n, x)\right| \leq e^{-t(|| n||+|x|)}
$$

The proof of Theorem 4.1 can be found in [1]. For convenience, we give a short proof in the Appendix.

Now we introduce the Cayley transformation for a self-adjoint operator.

Definition 4.2. Given a self-adjoint operator $A$ on Hilbert space $\mathbb{H}$, we call operator $(I-i A)(I+i A)^{-1}$ the Cayley transformation for operator $A$.

Clearly, $(I+i A)^{-1}$ is well defined and $(I-i A)(I+i A)^{-1}$ is a unitary operator.

Lemma 4.3 ([4, 13] $)$. If a vector $u \in \mathcal{P}_{d}$ satisfies equation (15), and $c=\left(1+i \lambda \Gamma_{0}\right) \varphi$, then $c \in \mathcal{P}_{d}$ and

$$
\left(1+i v^{-1}\right)\left(1-i v^{-1}\right)^{-1} c=\left(1-i \lambda \Gamma_{0}\right)\left(1+i \lambda \Gamma_{0}\right)^{-1} c .
$$

The converse is true. More concretely, if $c \in \mathcal{P}_{d}$ and satisfies (18), then the vector $\varphi=\left(1+i \lambda \Gamma_{0}\right)^{-1} c \in \mathcal{P}_{d}$ and satisfies equation (15).

It is easy to see that operator $\left(1+i v^{-1}\right)\left(1-i v^{-1}\right)^{-1}$ is multiplication by

$$
-e^{-2 \pi i(n \cdot \alpha+\theta)} \text {. }
$$

We extend the Fourier transform to a sequence $f_{n} \in \mathcal{P}_{d}$ in a distributional sense. More concretely, for a given sequence $f_{n} \in \mathcal{P}_{d}$, define the Fourier transform by the following equation:

$$
\hat{f}(y)=\sum_{n \in \mathbb{Z}^{d}} e^{-2 \pi i n \cdot y} f_{n} .
$$

By Fourier transformation, the equation (18) becomes

$$
q(y) \hat{c}(y)=e^{-2 \pi i \theta} \hat{c}(y+\alpha),
$$

where $q(y)=-\frac{1-i \lambda \hat{\Gamma}_{0}(y)}{1+i \lambda \hat{\Gamma}_{0}(y)}$. It is easy to see that $q(y)$ is analytic on $\mathbb{T}^{d}$, and $|q(y)|=1$ for $y \in \mathbb{R}^{d}$. We can rewrite

$$
q(y)=e^{-2 \pi i \zeta(y)},
$$

where $\zeta$ is analytic on $\mathbb{T}^{d}$. Notice that $\zeta$ only depends on $\lambda$ and $E$.

Next we will study the ergodicity of the analytical function. The proof is similar to the proof in 10 . 
Let $\frac{p_{n}}{q_{n}}$ be the continued fraction approximants to $\alpha \in \mathbb{R} \backslash \mathbb{Q}$. Then

$$
\forall 1 \leq k<q_{n+1}, \operatorname{dist}(k \alpha, \mathbb{Z}) \geq\left|q_{n} \alpha-p_{n}\right|,
$$

and

$$
\frac{1}{2 q_{n+1}} \leq \Delta_{n}:=\left|q_{n} \alpha-p_{n}\right| \leq \frac{1}{q_{n+1}} .
$$

We define an index $\beta(\alpha)$ for $\alpha \in \mathbb{R} \backslash \mathbb{Q}$,

$$
\beta(\alpha)=\limsup _{n \rightarrow \infty} \frac{\ln q_{n+1}}{q_{n}} .
$$

Lemma 4.4. Let $f: \mathbb{T}=\mathbb{R} / \mathbb{Z} \rightarrow \mathbb{R}$ be a real analytical function on the strip $\{z:|\Im z| \leq \rho\}$ and $\alpha \in \mathbb{R} \backslash \mathbb{Q}$. Suppose $\beta(\alpha)=0$. Then for any integer sequence $\left\{j_{k}\right\}$ such that

$$
\lim _{k \rightarrow \infty}\left\|j_{k} \alpha\right\|_{\mathbb{R} / \mathbb{Z}}=0,
$$

we have for $x \in\left\{z:|\Im z| \leq \frac{\rho}{2}\right\}$ uniformly,

$$
\lim _{k \rightarrow \infty}\left(\sum_{j=0}^{j_{k}-1} f(x+j \alpha)-j_{k} \int_{\mathbb{T}} f(x) d x\right)=0 .
$$

Proof. By the assumption, one has $\left|f_{k}\right| \leq C e^{-2 \pi \rho|k|}$, where $f_{k}$ is the Fourier coefficients of $f(x)$,

$$
\sum_{m=0}^{j_{k}-1} f(x+m \alpha)-j_{k} \int_{\mathbb{T}} f(x)=\sum_{j \neq 0} \frac{1-e^{-2 \pi i j j_{k} \alpha}}{1-e^{-2 \pi i j \alpha}} f_{j} e^{-2 \pi j x} .
$$

By the fact $\left\|j_{k} \alpha\right\|_{\mathbb{R} / \mathbb{Z}} \rightarrow 0$, it suffices to show

$$
\sum_{j \neq 0} \sup _{k}\left|\frac{1-e^{-2 \pi i j j_{k} \alpha}}{1-e^{-2 \pi i j \alpha}} f_{j}\right| e^{\rho \pi|j|}<\infty .
$$

By (21), (22) and $\beta(\alpha)=0$, one has

$$
\|j \alpha\|_{\mathbb{R} / \mathbb{Z}} \geq c e^{-\frac{\rho}{4}|j|} .
$$

Thus

$$
\begin{aligned}
\left|\frac{1-e^{-2 \pi i j j_{k} \alpha}}{1-e^{-2 \pi i j \alpha}} f_{j}\right| & \leq C e^{\frac{\rho}{4}|j|} e^{-2 \pi \rho|j|} \\
& \leq C e^{-\frac{3 \pi \rho}{2}|j|} .
\end{aligned}
$$

This implies (25).

Now we are concerned with the case $\beta(\alpha)>0$. By the definition, there exists a sequence $\left\{q_{n_{k}}\right\}$ such that

$$
q_{n_{k}+1} \geq e^{\frac{3}{4} \beta q_{n_{k}}} .
$$

Lemma 4.5. Let $f: \mathbb{T}=\mathbb{R} / \mathbb{Z} \rightarrow \mathbb{R}$ be a real analytical function on the strip $\{z:|\Im z| \leq \rho\}$. Given $\alpha \in \mathbb{R} \backslash \mathbb{Q}$, suppose $\beta(\alpha)>0$ and let $\bar{\rho}=\frac{1}{30} \inf \{\rho, \beta\}$. Let

$$
\left\{t_{m}\right\}_{m=1}^{\infty}=\left\{q_{n_{k}}, 2 q_{n_{k}}, 3 q_{n_{k}}, \cdots, \ell_{k} q_{n_{k}}\right\}_{k=1}^{\infty}
$$


where $q_{n_{k}}$ is given by (26) and $\ell_{k}=\left\lfloor e^{\bar{\rho} q_{n_{k}}}\right\rfloor \mathbb{1}$ Then for $x \in\left\{z:|\Im z| \leq \frac{\bar{\rho}}{2}\right\}$ uniformly,

$$
\lim _{m \rightarrow \infty}\left(\sum_{j=0}^{t_{m}-1} f(x+j \alpha)-t_{m} \int_{\mathbb{T}} f(x) d x\right)=0 .
$$

Proof. First, we have

$$
\sum_{j=0}^{t_{m}-1} f(x+j \alpha)-t_{m} \int_{\mathbb{T}} f(x)=\sum_{j \neq 0} \frac{1-e^{-2 \pi i j t_{m} \alpha}}{1-e^{-2 \pi i j \alpha}} f_{j} e^{-2 \pi j x} .
$$

By (22) and (26), one has

$$
\begin{aligned}
\left\|t_{m} \alpha\right\|_{\mathbb{R} / \mathbb{Z}} & \leq\left\lfloor e^{\bar{\rho} q_{n_{k}}}\right\rfloor\left\|q_{n_{k}} \alpha\right\|_{\mathbb{R} / \mathbb{Z}} \\
& \leq C e^{\bar{\rho} q_{n_{k}}} e^{-3 \bar{\rho} q_{n_{k}}} \\
& \leq C e^{-2 \bar{\rho} q_{n_{k}}} .
\end{aligned}
$$

This means $\left\|t_{m} \alpha\right\|_{\mathbb{R} / \mathbb{Z}} \rightarrow 0$; then it suffices to show

$$
\sum_{j \neq 0} \sup _{m}\left|\frac{1-e^{-2 \pi i j t_{m} \alpha}}{1-e^{-2 \pi i j \alpha}} f_{j}\right| e^{\pi \bar{\rho}|j|}<\infty .
$$

Fix some $t_{m}=\ell q_{n_{k}}$ with $0<\ell \leq e^{\bar{\rho} q_{n_{k}}}$.

Case 1. $|j|=q_{n}$ for some $n$.

If $n \leq n_{k}-1$, by (22) and (26), one has

$$
\begin{aligned}
\left|\frac{1-e^{-2 \pi i j t_{m} \alpha}}{1-e^{-2 \pi i j \alpha}} f_{j}\right| & \leq C|j| \ell \frac{\left\|q_{n_{k}} \alpha\right\|_{\mathbb{R} / \mathbb{Z}}}{\left\|q_{n} \alpha\right\|_{\mathbb{R} / \mathbb{Z}}}\left|f_{j}\right| \\
& \leq C|j| \ell \frac{q_{n+1}}{q_{n_{k}+1}}\left|f_{j}\right| \\
& \leq C|j| e^{\bar{\rho} q_{n_{k}}} \frac{q_{n_{k}}}{q_{n_{k}+1}}\left|f_{j}\right| \leq e^{-2 \pi \bar{\rho}|j|} .
\end{aligned}
$$

If $n=n_{k}$, by (22) and (26), one has

$$
\begin{aligned}
\left|\frac{1-e^{-2 \pi i j t_{m} \alpha}}{1-e^{-2 \pi i j \alpha}} f_{j}\right| & \leq C|j| \ell \frac{\left\|q_{n_{k}} \alpha\right\|_{\mathbb{R} / \mathbb{Z}}}{\left\|q_{n_{k}} \alpha\right\|_{\mathbb{R} / \mathbb{Z}}}\left|f_{j}\right| \\
& \leq C|j| \ell\left|f_{j}\right| \\
& \leq e^{-2 \pi \bar{\rho}|j|} .
\end{aligned}
$$

If $n \geq n_{k}+1$, by (22) again, one has

$$
\begin{aligned}
\left|\frac{1-e^{-2 \pi i j t_{m} \alpha}}{1-e^{-2 \pi i j \alpha}} f_{j}\right| & \leq C \frac{t_{m}|| j \alpha \|_{\mathbb{R} / \mathbb{Z}}}{\|j \alpha\|_{\mathbb{R} / \mathbb{Z}}} \mid \\
& \leq f^{-2 \pi \bar{\rho}|j|} .
\end{aligned}
$$

Case 2. $q_{n}<|j|<q_{n+1}$ for some $n$.

If $|j| \geq \frac{1}{\bar{\rho}} \ln q_{n+1}$, by (21) and (22), we have

$$
\begin{aligned}
\left|\frac{1-e^{-2 \pi i j t_{m} \alpha}}{1-e^{-2 \pi i j \alpha}} f_{j}\right| & \leq C q_{n+1}\left|f_{j}\right| \\
& \leq C e^{-2 \pi \bar{\rho}|j|} .
\end{aligned}
$$

\footnotetext{
${ }^{1}\lfloor\ell\rfloor$ denotes the smallest integer not exceeding $\ell$.
} 
If $q_{n}<|j|<q_{n+1}$ and $|j| \leq \frac{1}{\bar{\rho}} \ln q_{n+1}$, let $|j|=\ell^{\prime} q_{n}+j_{0}$ with $\left|\ell^{\prime}\right| \leq \frac{\ln q_{n+1}}{q_{n} \bar{\rho}}$ and $0 \leq\left|j_{0}\right|<q_{n^{\prime}}$. Assume $j_{0} \neq 0$; then by (21) and (22), one has

$$
\begin{aligned}
\|j \alpha\|_{\mathbb{R} / \mathbb{Z}} & \geq \Delta_{n-1}-\ell^{\prime} \Delta_{n} \\
& \geq \frac{1}{C q_{n}} .
\end{aligned}
$$

Thus we have

$$
\begin{aligned}
\left|\frac{1-e^{-2 \pi i j t_{m} \alpha}}{1-e^{-2 \pi i j \alpha}} f_{j}\right| & \leq C q_{n}\left|f_{j}\right| \\
& \leq e^{-2 \pi \bar{\rho}|j|} .
\end{aligned}
$$

Assume $j_{0}=0$; then $|j|=\ell^{\prime} q_{n}$ with $\left|\ell^{\prime}\right| \leq \frac{\ln q_{n+1}}{q_{n} \bar{\rho}}$. Applying the same proof as in Case 1, we also have

$$
\left|\frac{1-e^{-2 \pi i j t_{m} \alpha}}{1-e^{-2 \pi i j \alpha}} f_{j}\right|<e^{-2 \pi \bar{\rho}|j|}
$$

The estimate (28) is easy to obtain from the above cases.

The proof of Theorem 1.4. By Theorem 1.1 and Remark 1.5, it suffices to show that in regime $\mathbb{R} \backslash\left[-c_{d}, c_{d}\right] \quad(d=1)$, the spectral measures of the surface Maryland model are supported on a Hausdorff dimension zero set (we only prove the case for operator $H)$. Let

$B=\{E: E \in \mathbb{R} \backslash[-4,4]$ such that equation $H \psi=E \psi$ has a polynomial bounded solution $\}$.

By the Sch'nol Theorem [3], $B$ is a support of the spectral measures restriction on $(-\infty, \infty) \backslash[-4,4]$. We will prove that $B$ is a Hausdorff dimension zero set, which implies the theorem directly. Below are the details.

Let $E \in B$; then there exists a polynomial bounded solution of $\psi_{n, x}$ of equation $H \psi=E \psi$. By Theorem 4.1 and Lemma 4.3, we have (using (19))

$$
e^{-2 \pi i \zeta(y)} \hat{c}(y)=e^{-2 \pi i \theta} \hat{c}(y+\alpha),
$$

where $\hat{c}(y)$ is the Fourier transformation of a polynomially bounded vector $c \in$ $\ell^{2}(\mathbb{Z})$.

Case 1. $\beta(\alpha)=0$.

For any sequence $\left\{j_{k}\right\}$ such that $\lim _{k \rightarrow \infty}\left\|j_{k} \alpha\right\|_{\mathbb{R} / \mathbb{Z}}=0$ and iterate (30) $j_{k}$ times. We have

$$
e^{-2 \pi i \sum_{j=0}^{j_{k}-1} \zeta(y+j \alpha)} \hat{c}(y)=e^{-2 \pi i j_{k} \theta} \hat{c}\left(y+j_{k} \alpha\right) .
$$

Notice that $\hat{c}\left(y+j_{k} \alpha\right) \rightarrow \hat{c}(y)$ and combine with (24); then one has

$$
\lim _{n \rightarrow \infty}\left\|j_{k}\left(\zeta_{0}(E)-\theta\right)\right\|_{\mathbb{R} / \mathbb{Z}}=0,
$$

where $\zeta_{0}=\int_{\mathbb{R} / \mathbb{Z}} \zeta(y) d y$. By the arbitrary $j_{k}$, we must have

$$
\zeta_{0}(E) \in \theta+\alpha \mathbb{Z}+\mathbb{Z} .
$$

Notice that $\zeta_{0}(E)$ (non-constance) is analytical for $E \in(-\infty, \infty) \backslash[-4,4]$; then $B$ only contains a countable set. This means the Hausdorff dimension of $B$ is zero. 
Case 2. $\beta(\alpha)>0$.

By the definition of $\zeta(y), \zeta(y)$ is analytical on the strip $\{z:|\Im z| \leq \rho(E)\}$, where $\rho(E)>0$ is uniform with respect to $|E|-4$. Let $\left\{t_{m}\right\}$ be given by Lemma 4.5 and iterate (30) $t_{m}$ times. We have

$$
e^{-2 \pi i \sum_{j=0}^{t_{m}-1} \zeta(y+j \alpha)} \hat{c}(y)=e^{-2 \pi i t_{m} \theta} \hat{c}\left(y+t_{m} \alpha\right) .
$$

By (27) and the fact $\left\|t_{m} \alpha\right\|_{\mathbb{R} / \mathbb{Z}} \rightarrow 0$, we get

$$
\lim _{m \rightarrow \infty}\left\|t_{m}\left(\zeta_{0}(E)-\theta\right)\right\|_{\mathbb{R} / \mathbb{Z}}=0 .
$$

This implies for large $k$,

$$
\left\|q_{n_{k}}\left(\zeta_{0}(E)-\theta\right)\right\|_{\mathbb{R} / \mathbb{Z}} \leq e^{-\frac{\bar{\rho}}{2} q_{n_{k}}}
$$

where $\bar{\rho}$ has a uniformly non-zero lower bound depending on $|E|-4$ and $\beta(\alpha)$.

It is easy to check the set $\left\{x \in \mathbb{R}:\left\|q_{n_{k}} x\right\|_{\mathbb{R} / \mathbb{Z}} \leq e^{-\frac{\bar{\rho}}{2} q_{n_{k}}}\right.$ for large $\left.k\right\}$ is a Hausdorff measure zero set. In addition $\zeta_{0}(E)$ is analytical for $E \in(-\infty, \infty) \backslash[-4,4]$. We know $B$ is also a Hausdorff measure zero set.

For operator $H^{+}$, we only need to replace the operator $G_{0}, \Gamma_{0}$, etc., with $G_{0}^{+}$, $\Gamma_{0}^{+}$, etc. To avoid repetition, we omit the proof.

\section{APPENDix A.}

The proof of Theorem 4.1. If $\varphi_{n}$ is non-zero polynomially bounded and solves equation (15), it is easy to compute directly that $\psi_{n, x}$ given by equation (16) is non-zero polynomially bounded and solves equation (11).

To the contrary, suppose $\psi_{n, x}$ is non-zero polynomially bounded and solves equation (11). This implies

$$
\left(\left(H_{0}-E I\right) \psi\right)_{(n, x)}=-\delta(x) \lambda v(n) \psi_{n, x} .
$$

Setting $G_{0}=\left(H_{0}-E I\right)^{-1}$ on equation (31), one has

$$
\psi_{(n, x)}=-\sum_{\eta \in \mathbb{Z}^{d}} G_{0}(n-\eta, x) \lambda v(\eta) \psi_{\eta, 0} .
$$

Thus $\psi_{\eta, 0}$ is a non-zero vector, and letting $x=0$ in (32), we obtain

$$
\psi_{(n, 0)}=-\sum_{\eta \in \mathbb{Z}^{d}} \Gamma_{0}(n-\eta) \lambda v(\eta) \psi_{\eta, 0}
$$

This yields that $\varphi_{n}$ given by (17) solves equation (15) and is non-zero polynomially bounded. We complete the proof of Theorem 4.1 .

\section{ACKNOWLEDGMENTS}

This research was partially supported by NSF DMS-1401204. The author would like to thank Svetlana Jitomirskaya for drawing attention to the problem and the inspiring discussions on this subject. 


\section{REFERENCES}

[1] F. Bentosela, Ph. Briet, and L. Pastur, On the spectral and wave propagation properties of the surface Maryland model, J. Math. Phys. 44 (2003), no. 1, 1-35, DOI 10.1063/1.1521798. MR.1946689

[2] F. Bentosela, Ph. Briet, and L. Pastur, Spectral analysis of the generalized surface Maryland model, Algebra i Analiz 16 (2004), no. 6, 1-27, DOI 10.1090/S1061-0022-05-00884-8; English transl., St. Petersburg Math. J. 16 (2005), no. 6, 923-942. MR2117447

[3] Ju. M. Berezans'kiū, Expansions in eigenfunctions of selfadjoint operators, Translated from the Russian by R. Bolstein, J. M. Danskin, J. Rovnyak and L. Shulman. Translations of Mathematical Monographs, Vol. 17, American Mathematical Society, Providence, R.I., 1968. MR.0222718

[4] H. Cycon, R. Froese, W. Kirsch, and B. Simon, Schrödinger operators with applications to quantum mechanics and global geometry, 1987.

[5] A. L. Figotin and L. A. Pastur, An exactly solvable model of a multidimensional incommensurate structure, Comm. Math. Phys. 95 (1984), no. 4, 401-425. MR767188

[6] Shmuel Fishman, D. R. Grempel, and R. E. Prange, Chaos, quantum recurrences, and Anderson localization, Phys. Rev. Lett. 49 (1982), no. 8, 509-512, DOI 10.1103/PhysRevLett.49.509. MR669169

[7] Vojkan Jakšić and Stanislav Molchanov, On the spectrum of the surface Maryland model, Lett. Math. Phys. 45 (1998), no. 3, 189-193, DOI 10.1023/A:1007579806383. MR.1641176

[8] Vojkan Jakšić and Stanislav Molchanov, On the surface spectrum in dimension two, Helv. Phys. Acta 71 (1998), no. 6, 629-657. MR.1669046

[9] Vojkan Jakšić and Stanislav Molchanov, Localization of surface spectra, Comm. Math. Phys. 208 (1999), no. 1, 153-172, DOI 10.1007/s002200050752. MR1729882

[10] S. Jitomirskaya and W. Liu, Arithmetic spectral transitions for the Maryland model, Preprint, 2014.

[11] B. A. Khoruzhenko and L. A. Pastur, The localization of surface states: an exactly solvable model, Physics Reports, 288(1):109-126, 1997.

[12] M. Reed and B. Simon, Methods of Modern Mathematical Physics: Vol.: 1.: Functional Analysis, Academic press, 1972.

[13] Barry Simon, Almost periodic Schrödinger operators. IV. The Maryland model, Ann. Physics 159 (1985), no. 1, 157-183, DOI 10.1016/0003-4916(85)90196-4. MR776654

School of Mathematical Sciences, Fudan University, Shanghai 200433, People's RePUBLIC OF CHINA

E-mail address: liuwencai1226@gmail.com

Current address: Department of Mathematics, University of California, Irvine, California 92697-3875 